\title{
Endoscopic Resection of Cervical Vagal Nerve Schwannoma
}

\author{
Xi Yang, MM, Junlan Liu, MB, Linjun Fan, PhD \\ Breast Disease Center, Southwest Hospital of The Third Military Medical University, Chongqing China (all authors).
}

\begin{abstract}
Introduction: Currently, surgical resection is the only effective treatment for schwannoma. Long-term compression may bring irreversible damage to nerves. The conventional surgery for cervical vagal nerve schwannoma often produces a visible scar on the neck, an unsatisfactory cosmetic outcome.

Case Description: We performed an endoscopic resection of vagal nerve schwannoma on the right side of the neck in a 15-year-old male patient. Three incisions were made in the bilateral areolae and right axilla, and trocars were inserted along the line from the incision to the right supraclavicular fossa. An ultrasonic scalpel and electrocoagulation hook was used to dissect and remove the tumor in the deep face of the sternocleidomastoid and strap muscles.
\end{abstract}

Results: The vagal nerve schwannoma was completely excised with no significant intraoperative bleeding. The operation time was 45 minutes. The postoperative recovery was uneventful, and no recurrence was found in the 3-year follow-up.

Conclusion: Endoscopic resection via the bilateral areolae and axilla approach is a safe and feasible surgery for cervical vagal nerve schwannoma, providing a covert incision and satisfactory cosmetic outcome.

Key Words: Endoscope, Neck, Resection, Vagal nerve schwannoma.

Citation Yang X, Liu J, Fan L. Endoscopic resection of cervical vagal nerve schwannoma. CRSLS e2015.00109. DOI: 10.4293/CRSLS.2015.00109.

Copyright $\odot 2016$ by SLS, Society of Laparoendoscopic Surgeons. This is an open-access article distributed under the terms of the Creative Commons Attribution-Noncommercial-ShareAlike 3.0 Unported license, which permits unrestricted noncommercial use, distribution, and reproduction in any medium, provided the original author and source are credited.

This work was supported by Clinical Research Project 2010XLc010 of the Third Military Medical University.

Address correspondence to: Linjun Fan, PhD, Breast Disease Center, Southwest Hospital of The Third Military Medical University, Chongqing 400038, China.

Telephone: +86-13389668035, Fax: +8623-68765277; E-mail: flj212@medmail.com.cn

\section{INTRODUCTION}

Schwannomas, originating from neurilemma cells, can form in cranial, peripheral, and autonomic nerves. Schwannoma in the head and neck occurs with moderate frequency, accounting for $45 \%$ of cases. ${ }^{1}$ Vagal nerve schwannoma is rarely seen in clinics, ${ }^{2}$ mostly located in the carotid sheath. It often presents as a well-circumscribed, encapsulated, slow-growing, asymptomatic tumor in the carotid triangle. ${ }^{3}$ If the mass grows to a certain size, it can compress adjacent organs and result in neurological dysfunction (such as coughing and choking when a drink is consumed, and hoarseness); therefore, surgical resection is necessary in this situation.

Conventional surgery to excise cervical vagal nerve schwannoma, including the transcervical, transcondylar, cervical transmastoid, and infratemporal fossa approaches, usually leaves visible scars on the neck. ${ }^{4}$ Considering that such tumors often occur in young adults, who are deeply concerned about their appearance, those visible scars may become a cosmetic problem. In August 2012, we treated one patient with a vagal nerve schwannoma on the right side of the neck with endoscopic resection via the bilateral areolae and right axilla approach and obtained satisfactory postoperative recovery. No vagal nerve dysfunction was found during follow-up visits.

\section{CASE REPORT}

A 15-year-old boy with a mass on the right side of his neck for over 2 years was admitted to our department. The patient had had the $3.0 \times 2.0-\mathrm{cm}$ mass for 1 year. After 1 year, the mass had gradually increased to $3.5 \times 2.5 \mathrm{~cm}$. The patient had no history of surgery or trauma; no neck pain or swelling; no hoarseness, drink cough, or parox- 
ysmal cough produced on palpating the mass; and no dysphagia, weight loss, fever, chills, or chest pain. A physical examination showed that the trachea was in the normal central position, with no jugular engorgement and a negative hepatojugular reflux sign. A $3.5 \times$ $2.5-\mathrm{cm}$ mass was palpated in the right carotid triangle. It moved during swallowing, with a clear margin and a smooth surface. There was no palpable mass on the left side of the neck, no thyroid enlargement, and no palpable cervical lymph nodes on either side of the neck. No abnormalities were found in routine blood tests, no liver or renal disorder, and no positive findings in $\mathrm{T} 3$, $\mathrm{T} 4$, and TSH tests.

Color Doppler ultrasonography (L12-5; Philips, Eindhoven, The Netherlands) indicated a $35.4 \times 25.7-\mathrm{mm}$ low-echo area at the root of the right side of the neck, lateral to the common carotid artery and posterior to the jugular vein, with clear margin and in intact capsule and several limited no-echo regions inside the mass. Color Doppler flow imaging demonstrated rich linear blood flow signals, and recorded the pulsatile flow spectrum, with Resistance Index $(R I)=0.47$ (Figure 1). Computed tomography (Somatom Emotion 16; Siemens, Forchheim, Germany) results showed a $3.5 \times 2.6-\mathrm{cm}$ nodular soft tissue shadowing on the right lobe of the thyroid, posterolateral to right carotid artery and posteromedial to right internal jugular vein, with uneven density enhancement and a clear margin. The mass was closely connected with the right carotid artery sheath, leading to the compression on adjacent soft tissue, whereas the tumor margin remained clear. A neurogenic tumor was suspected (Figure 2).

\section{Surgical Techniques}

After induction of general anesthesia, the patient was positioned with his shoulder elevated and pads were placed at his back to cause his neck to be extended. The surgical procedure followed our thyroid surgery approach, as previously described [5, 6]. First, the border of the mass, median of the anterior neck, the incision line, and the route of trocar insertion were marked on the skin surface (Figure 3). Then, three 0.5-0.8-cm-long incisions in the upper inner margins of the bilateral areolae and right axilla were made, and the trocars were inserted along the line from the incision to the right supraclavicular fossa. Three trocars met in the right supraclavicular fossa, and insufflation with $\mathrm{CO}_{2}$ was performed (to maintain a pressure of $6 \mathrm{~mm} \mathrm{Hg}$ ) to establish an endoscopic working space. The 2 incisions in the right axilla and areola served as operation ports, the left areola incision as the endo-

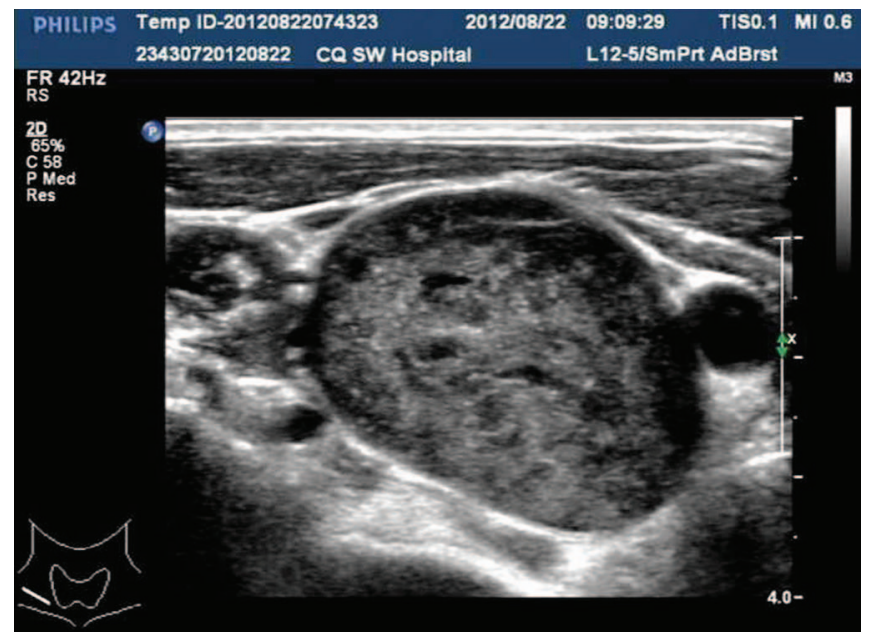

Figure 1. Color Doppler ultrasound images of the neck: a $35.4 \times$ 25.7-mm low-echo area with a clear margin was observed at the root of the right side of the neck, lateral to the common carotid artery and posterior to the jugular vein. The tumor was suspected to be coated.

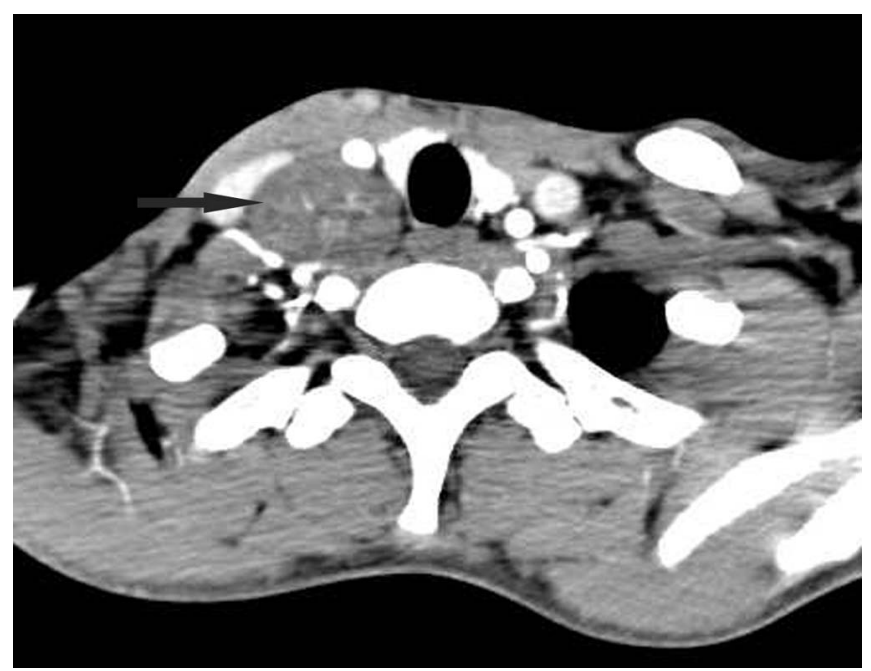

Figure 2. Computed tomographic image of the neck. A $3.5 \times$ 2.6-cm nodular soft tissue shadowing was observed on the right lobe of the thyroid (arrow), posterolateral to the right carotid artery, and posteromedial to the right internal jugular vein, with uneven density enhancement and a clear margin distinguished from the surrounding tissues. The adjacent structures were physically disabled and displaced because of compression.

scopic observation port (endoscope: $30^{\circ}, 10 \mathrm{~mm}$ in diameter; Olympus, Tokyo, Japan). Starting from the right supraclavicular fossa, the skin flap was dissected deep into the platysma along the surface of the right sternocleidomastoid muscle and strap muscle, up to the upper margin of the thyroid cartilage, right to the lateral border of the 


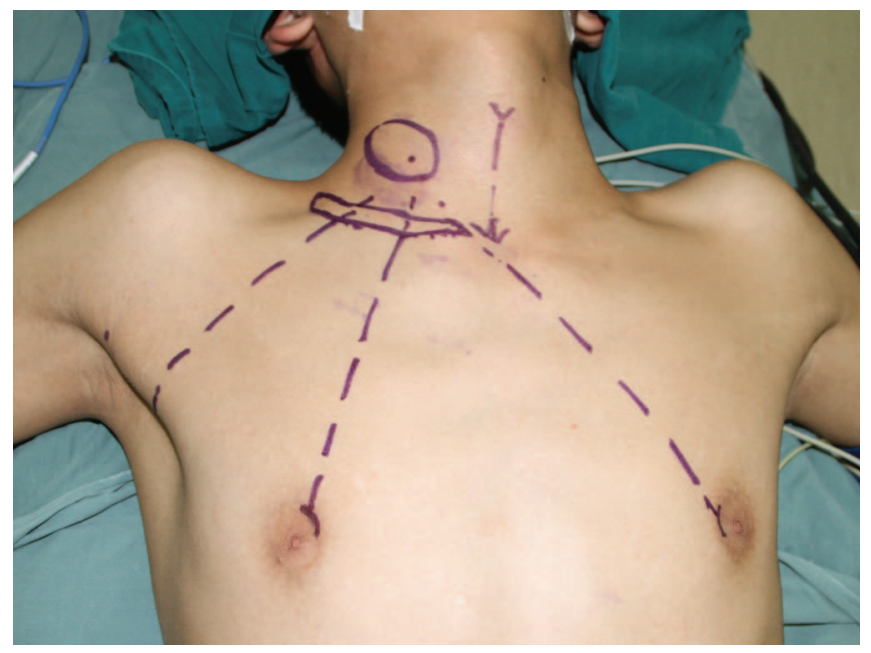

Figure 3. Before the operation, skin surface markings outline the border of the mass, the anterior median line of the neck, the incision line, and the routes of trocar insertion.

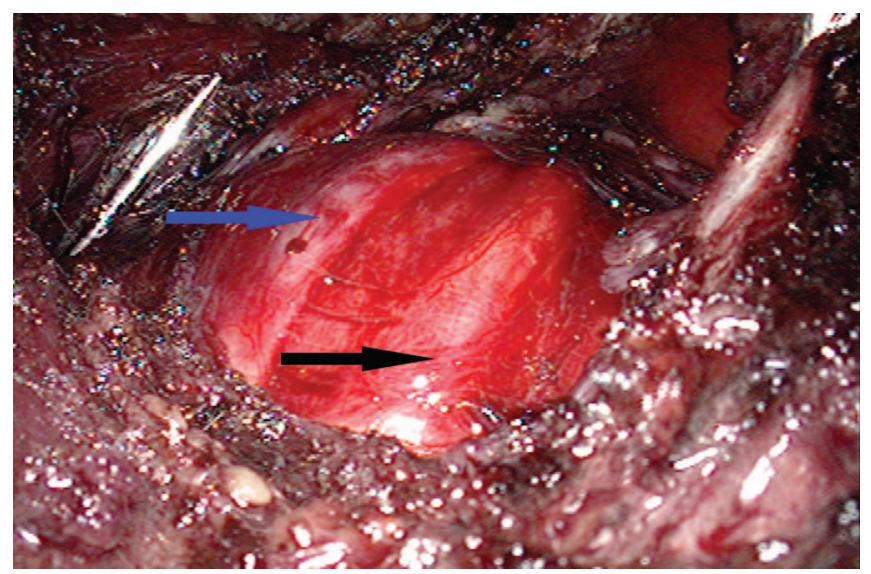

Figure 4. The right sternocleidomastoid and strap muscles were drawn apart to expose the mass. The internal jugular vein (blue arrow) and the vagal nerve (black arrow) were on the tumor surface, and the internal jugular vein was significantly compressed.

right sternocleidomastoid muscle, and medial to the midline of the neck. After the flap was separated to a predetermined depth, the right sternocleidomastoid and strap muscles were drawn to different sides to expose the mass. The internal jugular vein and the vagal nerve were on the tumor surface, and the internal jugular vein was obviously compressed (Figure 4). The common carotid artery, internal jugular vein, and vagal nerve were dissected and draw to different sides. The tumor was separated along the tumor capsule with an ultrasonic scalpel, its vascular pedicle was cut off, and finally it was resected completely. The surgical field was rinsed thoroughly with sterile distilled water to ensure that there was no active bleeding, a

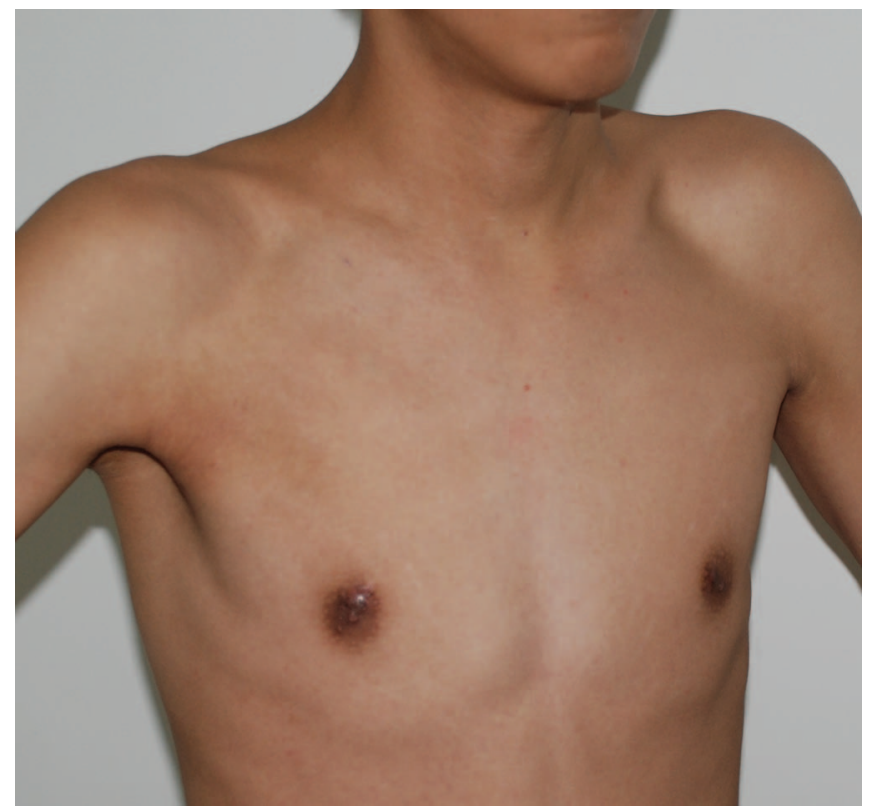

Figure 5. Postoperative appearance of the neck at the 1-year follow-up. The incisions were covert and the patient was very satisfied with the appearance of the neck and the incisions.

4.5-mm-diameter silicone drainage tube was placed in the surgical field, and the other end of the tube was pulled from the ipsilateral areola incision and fixed.

Operative time was 45 minutes, and there was no significant intraoperative bleeding. The amount of fluid drainage recorded was less than $10 \mathrm{~mL} 3$ days after the operation, and the drainage tube was removed. Pathology results confirmed right neck schwannoma. The patient was discharged 4 days after surgery and recovered well with no vagal nerve dysfunction. In a 3-year follow-up, no recurrence was reported, and the patient was very satisfied with the cosmetic result (Figure 5).

\section{DISCUSSION}

Endoscopic surgery has become increasingly popular because of the advantages of minimal trauma, quick recovery, and good cosmetic effect when compared with open surgery. Currently in cervical surgery, there are many studies focused on endoscopy for resection of thyroid and parathyroid masses,, $5-11$ but endoscopic resection of a mass that is not located in the thyroid or parathyroid is rarely reported. We performed an endoscopic excision of branchial cleft cyst via an incision far away from the neck (mammary areolae and axilla approach) and achieved a good cosmetic result. ${ }^{11}$ In the current study, the patient was a 15 -year-old boy, who 
requested an excellent aesthetic outcome. Because conventional surgery to remove a neck schwannoma (eg, a vagal nerve schwannoma) will leave a noticeable scar on the neck, we applied endoscopic surgery to resect the schwannoma deep below the surface of the neck through bilateral areolae and axilla incisions, resulting in no scars on the neck and fulfilling the cosmetic goals of the patient.

Vagal nerve schwannoma is located in the carotid sheath and is enveloped with the common carotid artery, the internal jugular vein, and vagal nerve. Thus, any surgical accident could damage these important structures. In this case, with sternocleidomastoid and strap muscles as anatomic landmarks, we dissected the tumor in the deep muscle surface layer by layer. The surgeon can easily identify the tumor, as well as its surrounding tissues, via the amplification effect of endoscopy and resect the tumor with an ultrasonic scalpel close to the capsule. Compared with conventional surgery, the endoscopic procedure causes a smaller range of thermal damage and injury to the surrounding tissues, given that it can protect the vagal nerve trunk and nerve function more precisely. The operative time was 45 minutes, with no significant blood loss. Therefore, a cervical mass that is not in the thyroid or parathyroid can also be resected by endoscopy in a safe, simple, and convenient manner.

In summary, the endoscopic resection via bilateral areolae and axilla approach is the treatment of choice for cervical vagal nerve schwannoma, with the advantages of convenient operative access, covert incisions, beneficial cosmetic outcome, less complication, quick recovery, and good prognosis.

\section{References:}

1. Das Gupta TK, Brasfield RD, Strong EW, Hajdu SI. Benign solitary Schwannomas (neurilemomas). Cancer. 1969;24(2): 355-66.
2. Gilmer-Hill HS, Kline DG. Neurogenic tumours of the cervical vagus nerve: report of four cases and review of the literature. Neurosurgery. 2000;46:1498-1503.

3. Chiofalo MG, Longo F, Marone U, et al. Cervical vagal schwannoma: a case report. Acta Otorhinolaryngol Ital. 2009; 29:33-35.

4. Chiun KC, Tang IP, Prepageran N, et al. An extensive cervical vagal nerve schwannoma: a case report. Med J Malaysia. 2012;67:342-344.

5. Fan LJ, Ming J, Huang DD, et al. Endoscopic near total thyroidectomy followed by radioiodine remnant ablation in differentiated thyroid carcinoma. J Otolaryngol Ophthalmol Shandong Univ. 2011;25:11-16.

6. Ming J, Fan Z, Gong Y, et al. Endoscopic excision of branchial cleft cyst in the neck using mammary areolae and axilla approach: a case report. Surg Laparosc Endosc Percutan Tech. 2012;22:e284-e287.

7. Koh YW, Kim JW, Lee SW, et al. Endoscopic thyroidectomy via a unilateral axillo-breast approach without gas insufflation for unilateral benign thyroid lesions. Surg Endosc 2009;23:20532060.

8. Park YL, Han WK, Bae WG. 100 cases of endoscopic thyroidectomy: breast approach. Surg Laparosc Endosc Percutan Tech. 2003;13:20-25.

9. Jung EJ, Park ST, Ha WS, et al. Endoscopic thyroidectomy using a gasless axillary approach. J Laparoendosc Adv Surg Tech A. 2007;17:21-25.

10. Yoon JH, Park CH, Chung WY. Gasless endoscopic thyroidectomy via an axillary approach: experience of 30 cases. Surg Laparosc Endosc Percutan Tech. 2006;16:226-231.

11. Sun Y, Cai H, Bai J, et al. Endoscopic total parathyroidectomy and partial parathyroid tissue autotransplantation for patients with secondary hyperparathyroidism: a new surgical approach. World J Surg 2009;33:1674-1679. 